\title{
Reaching a Consensus in Multi-Agent Systems: A Time Invariant Nonlinear Rule
}

\author{
${ }^{*}$ Mansoor Saburov ${ }^{1}$, Khikmat Saburov ${ }^{2}$ \\ ${ }^{1}$ International Islamic University Malaysia, Pahang, Malaysia \\ ${ }^{2}$ Stare Namesti 141, Sokolov, Czech Republic \\ *msaburov@gmail.com
}

\begin{abstract}
Multi-Agent Systems (MAS) have attracted more and more interest in recent years. Most researches in the study of discrete-time MAS, presented in the past few years, have considered linear cooperative rules. However, local interactions between agents are more likely to be governed by nonlinear rules. In this paper, we investigate the consensus of discrete-time MAS with time invariant nonlinear cooperative rules. Based on our presented nonlinear model, we show a consensus in the discrete-time MAS. Our model generalizes a classical time invariant De Groot model. It seems that, unlike a linear case, a consensus can be easily achieved a nonlinear case.
\end{abstract}

\section{Keywords: Multi-Agent System; Cooperative Control; Nonlinear Rule; Time Invariant; Nonlinear Consensus}

\section{Introduction}

Multi-Agent Systems (MAS) give a complete description for large-scale systems consisting of small subunits, called agents. The behavior of MAS is particularly interesting because the agents may fulfill certain tasks as a group, even in the individual agent does not know about the overall task. A lot of examples come from nature, such as schooling fishes or fireflies flashing in unison, see, e.g., Strogatz (2003). A collective behavior is also interesting for engineers when solving problems such as flocking (Fax \& Murray 2004, Jadbabaie et al., 2003, and Olfati-Saber, 2006) or synchronization (Jadbabaie et al., 2004, 2000). This is mainly due to its important applications, including the cooperative control of unmanned air vehicles (UAVs), autonomous underwater vehicles, congestion control in communication networks, swarms of autonomous vehicles or robots, autonomous formation fight, etc. In all cases, the goal is to control, a group of agents connected through a communication network to reach an agreement on certain quantities of interest. This problem is called the consensus problem. Many results have been achieved on this problem. Recent surveys of consensus and cooperation are given in (Olfati-Saber et al., 2007) and (Ren et al., 2008). Most researches in MAS consider only linear rule of exchanging information. However, many systems, such as for instance the well-known Kuramoto oscillator exhibit nonlinear, locally passive dynamics as discussed in (Kuramoto, 1984) and (Papachristodoulou \& Jadbabaie, 2005). Nonlinear consensus problems have been previously studied in (Bauso et al., 2006, Lin et al., 2007, and Moreau, 2005). In this paper, we shall present a time invariant nonlinear model of discrete-time MAS. Our model is a generalization of the classical time invariant DeGroot model (Berger, 1981 and DeGroot, 1974). Based on our time invariant model, we show a consensus in the discrete-time MAS. It seems that, unlike a linear case, a consensus can be easily achieved a nonlinear case.

\section{Methodology}

Historically, the idea of reaching consensus through repeated averaging was introduced by DeGroot (1974), for a structured, time-invariant, and synchronous environment. For reasons of self-exposition, it is convenient to provide a linear model for an estimate-modification process of a structured, time-invariant, and synchronous environment, which was presented in (Berger, 1981 and DeGroot, 1974). Let us consider a group of $m$ individuals, each of whom can specify his/her own subjective probability distribution for the given task. All individuals must act together as a team or committee. Let $\boldsymbol{x}_{\boldsymbol{i}}^{(0)}$ denote the subjective probability distribution that individual $\boldsymbol{i}$ assigns to the given task, for $\boldsymbol{i}=1, \cdots, \boldsymbol{m}$. It is clear that the subjective distributions $\boldsymbol{x}_{1}^{(0)}, \cdots, \boldsymbol{x}_{\boldsymbol{m}}^{(0)}$ will be based on the different backgrounds and different levels of expertise of the members of the group. We assume that if individual $i$ is informed of the distributions of each of the other members of the group, he/she might wish to revise his/her subjective distribution to accommodate this information. In the DeGroot's model (1974), it was assumed that when individual $i$ makes this revision, his/her revised distribution is a linear 
combination of the distributions $\boldsymbol{x}_{1}^{(0)}, \cdots, \boldsymbol{x}_{m}^{(0)}$. Therefore, after being informed of the subjective distributions of the other members of the group, individual $i$ revises his/her own subjective distribution from $\boldsymbol{x}_{i}^{(0)}$ to $\boldsymbol{x}_{i}^{(1)}=\sum_{j=1}^{m} \boldsymbol{p}_{i j} \boldsymbol{x}_{j}^{(0)}$. Let $\boldsymbol{P}$ denote the $\boldsymbol{m} \times \boldsymbol{m}$ matrix whose $(\boldsymbol{i}, \boldsymbol{j})$ th element is $\boldsymbol{p}_{i j}$. It is clear that $\boldsymbol{P}$ is a stochastic matrix since the elements are all non-negative and the rows sum to one. Let $\boldsymbol{x}^{(0)}=\left(\boldsymbol{x}_{1}^{(0)}, \cdots, \boldsymbol{x}_{\boldsymbol{m}}^{(0)}\right)$ and $\boldsymbol{x}^{(1)}=\left(\boldsymbol{x}_{1}^{(1)}, \cdots, \boldsymbol{x}_{\boldsymbol{m}}^{(1)}\right)$ be vectors. Then the vector of revised subjective distributions can be written as $\boldsymbol{x}^{(1)}=\boldsymbol{P x}^{(0)}$. The process continues in this way. Let $\boldsymbol{x}^{(\boldsymbol{n})}=\left(\boldsymbol{x}_{1}^{(\boldsymbol{n})}, \boldsymbol{x}_{2}^{(\boldsymbol{n})}, \ldots, \boldsymbol{x}_{\boldsymbol{m}}^{(\boldsymbol{n})}\right)$ denote the subjective distribution of MAS after $\boldsymbol{n}$ revisions controlled by a linear rule. Then $\boldsymbol{x}^{(\boldsymbol{n})}=\boldsymbol{P} \boldsymbol{x}^{(\boldsymbol{n}-1)}=\boldsymbol{P}^{n} \boldsymbol{x}^{(0)}$. We say that a consensus is reached if and only if all $\boldsymbol{m}$ components of $\boldsymbol{x}^{(\boldsymbol{n})}$ converge to the same limit as $\boldsymbol{n} \rightarrow \infty$.

In this paper, we shall consider a nonlinear model for an estimate-modification process of a structured, time invariant, and synchronous environment. Namely, we suppose that every individual makes a revision of his/her subjective distribution as a nonlinear combination of the previous distributions. More precisely, in our nonlinear model, after being informed of the subjective distributions of the other members of the group, individual $i$ revises his/her own subjective distribution from $\boldsymbol{x}_{i}^{(0)}$ to $\boldsymbol{x}_{i}^{(1)}=\sum_{j, k=1}^{m} \boldsymbol{p}_{i j k} \boldsymbol{x}_{j}^{(0)} \boldsymbol{x}_{\boldsymbol{k}}^{(0)}$ , where $\boldsymbol{P}=\left(\boldsymbol{p}_{i j k}\right)_{i, j, k=1}^{\boldsymbol{m}}$ is a triple stochastic cubic matrix with non-negative entries i.e.,

$$
\boldsymbol{p}_{i j k} \geq 0, \sum_{i=1}^{m} \boldsymbol{p}_{i j k}=\sum_{j=1}^{m} \boldsymbol{p}_{i j k}=\sum_{k=1}^{m} \boldsymbol{p}_{i j k}=1,
$$

for any $\boldsymbol{i}, \boldsymbol{j}, \boldsymbol{k}=\overline{1, \boldsymbol{m}}$.

In this process, the above revision is iterated. It is assumed that after individual $\boldsymbol{i}$ is informed of the revised subjective distributions, $\boldsymbol{x}_{1}^{(1)}, \cdots, \boldsymbol{x}_{\boldsymbol{m}}^{(1)}$ of the members of the group, he/she revises his/her subjective distribution from $\boldsymbol{x}_{i}^{(1)}$ to $\boldsymbol{x}_{i}^{(2)}=\sum_{j, k=1}^{m} \boldsymbol{p}_{i j k} \boldsymbol{x}_{j}^{(1)} \boldsymbol{x}_{k}^{(1)}$. The process continues in this way. Let $\boldsymbol{x}^{(\boldsymbol{n})}=\left(\boldsymbol{x}_{1}^{(\boldsymbol{n})}, \boldsymbol{x}_{2}^{(\boldsymbol{n})}, \ldots, \boldsymbol{x}_{\boldsymbol{m}}^{(\boldsymbol{n})}\right)$ denote the subjective distribution of MAS after $\boldsymbol{n}$ revisions controlled by a nonlinear rule.

Let $\quad \boldsymbol{S}^{\boldsymbol{m}-1}=\left\{\boldsymbol{x} \in \boldsymbol{R}^{\boldsymbol{m}}: \sum_{i=1}^{m} \boldsymbol{x}_{\boldsymbol{i}}=1, \boldsymbol{x}_{\boldsymbol{i}} \geq 0 \forall \boldsymbol{i}=\overline{1, \boldsymbol{m}}\right\} \quad$ be $\quad$ a $\quad$ simple. Every subjective distribution $\boldsymbol{x}^{(\boldsymbol{n})}=\left(\boldsymbol{x}_{1}^{(\boldsymbol{n})}, \boldsymbol{x}_{2}^{(\boldsymbol{n})}, \ldots, \boldsymbol{x}_{\boldsymbol{m}}^{(\boldsymbol{n})}\right)$ of MAS, after $\boldsymbol{n}$ revisions, belongs the simplex.

Let us define a nonlinear rule (operator) $V: S^{m-1} \rightarrow S^{m-1}$ as follows

$$
\boldsymbol{V} \boldsymbol{x}=\left(\sum_{i, j=1}^{m} \boldsymbol{p}_{i j 1} \boldsymbol{x}_{i} \boldsymbol{x}_{j}, \sum_{i, j=1}^{m} \boldsymbol{p}_{i j 2} \boldsymbol{x}_{i} \boldsymbol{x}_{j}, \cdots, \sum_{i, j=1}^{m} \boldsymbol{p}_{i j m} \boldsymbol{x}_{i} \boldsymbol{x}_{j}\right)
$$

where $\quad \boldsymbol{p}_{i j k} \geq 0, \quad \sum_{i=1}^{m} \boldsymbol{p}_{i j k}=\sum_{j=1}^{m} \boldsymbol{p}_{i j k}=\sum_{k=1}^{m} \boldsymbol{p}_{i j k}=1$, for any $\boldsymbol{i}, \boldsymbol{j}, \boldsymbol{k}=\overline{1, \boldsymbol{m}}$. In this case,

$\boldsymbol{x}^{(n)}=\boldsymbol{V}\left(\boldsymbol{x}^{(n-1)}\right)=\boldsymbol{V}^{\circ \boldsymbol{n}}\left(\boldsymbol{x}^{(0)}\right)$

In the nonlinear case, we say that MAS is reaching a consensus, if $\boldsymbol{x}^{(\boldsymbol{n})}$ converges to the center $\boldsymbol{C}=\left(\frac{1}{\boldsymbol{m}}, \frac{1}{\boldsymbol{m}}, \ldots, \frac{1}{\boldsymbol{m}}\right)$ of the simplex $\boldsymbol{S}^{\boldsymbol{m}-1}$. In order to check for reaching a consensus, we should show that the trajectory of the operator (1) converges to the center $C=\left(\frac{1}{m}, \frac{1}{m}, \ldots, \frac{1}{m}\right)$. The operator (1) is 
called a quadratic stochastic operator (Ganikhodzhaev et. al., 2012, Saburov, 2007, 2012, 2013).

\section{Results and Discussion}

In this section, we shall study dynamics of the operator (1). Let us state some mathematical results.

Theorem Let $\boldsymbol{V}: \boldsymbol{S}^{\boldsymbol{m}-1} \rightarrow \boldsymbol{S}^{\boldsymbol{m}-1}$ be a quadratic stochastic operator given by (1). Then the trajectory $\left\{\boldsymbol{x}^{(\boldsymbol{n})}\right\}$ of the operator $\boldsymbol{V}$ starting from an initial point $\boldsymbol{x}^{(0)} \in \operatorname{int} \boldsymbol{S}^{\boldsymbol{m}-1}$ converges to the center $\boldsymbol{C}=\left(\frac{1}{\boldsymbol{m}}, \frac{1}{\boldsymbol{m}}, \ldots, \frac{1}{\boldsymbol{m}}\right)$ of the simplex $\boldsymbol{S}^{\boldsymbol{m}-1}$.

Let us provide a sketch of the proof of Theorem. It is clear that the operator $V: S^{m-1} \rightarrow S^{m-1}$ given by (1) can be written as follows

$$
\boldsymbol{V} \boldsymbol{x}=\left(\left(\boldsymbol{P}_{1} \boldsymbol{x}, \boldsymbol{x}\right),\left(\boldsymbol{P}_{2} \boldsymbol{x}, \boldsymbol{x}\right), \cdots,\left(\boldsymbol{P}_{\boldsymbol{m}} \boldsymbol{x}, \boldsymbol{x}\right)\right)(2)
$$

where $\boldsymbol{P}_{\boldsymbol{k}}=\left(\boldsymbol{p}_{i j k}\right)_{i, j=1}^{\boldsymbol{m}}$ is a doubly stochastic matrix for any $\boldsymbol{k}=\overline{1, \boldsymbol{m}}$.

$$
\begin{aligned}
& \text { It follows from (2) that } \\
& \boldsymbol{V} \boldsymbol{x}=\boldsymbol{P}_{\boldsymbol{x}} \boldsymbol{x}
\end{aligned}
$$

where $\boldsymbol{P}_{\boldsymbol{x}}$ is a matrix depending on $\boldsymbol{x}$ and defined as $\boldsymbol{P}_{\boldsymbol{x}}=\left(\left(\boldsymbol{P}_{\boldsymbol{k}} \boldsymbol{x}\right)_{\boldsymbol{i}}\right)_{\boldsymbol{k}, \boldsymbol{i}=1}^{\boldsymbol{m}}$ i.e., a vector $\boldsymbol{P}_{\boldsymbol{k}} \boldsymbol{x}$ is a $\boldsymbol{k}$ - row vector of a matrix $\boldsymbol{P}_{\boldsymbol{x}}$. More precisely, we have that $\left(\boldsymbol{P}_{\boldsymbol{k}} \boldsymbol{x}\right)_{i}=\sum_{j=1}^{m} \boldsymbol{p}_{i j k} \boldsymbol{x}_{j}$ Let us show that $\boldsymbol{P}_{x}$ a doubly stochastic matrix for any $\boldsymbol{x} \in \boldsymbol{S}^{\boldsymbol{m}-1}$. Indeed, one has that

$$
\begin{aligned}
& \sum_{i=1}^{m}\left(\boldsymbol{P}_{k} \boldsymbol{x}\right)_{i}=\sum_{i=1}^{m}\left(\sum_{j=1}^{m} \boldsymbol{p}_{i j k} \boldsymbol{x}_{j}\right)=\sum_{j=1}^{m}\left(\sum_{i=1}^{m} \boldsymbol{p}_{i j k}\right) \boldsymbol{x}_{j}=\sum_{j=1}^{m} \boldsymbol{x}_{j}=1 \\
& \sum_{k=1}^{m}\left(\boldsymbol{P}_{k} \boldsymbol{x}\right)_{i}=\sum_{k=1}^{m}\left(\sum_{j=1}^{m} \boldsymbol{p}_{i j k} \boldsymbol{x}_{j}\right)=\sum_{j=1}^{m}\left(\sum_{k=1}^{m} \boldsymbol{p}_{i j k}\right) \boldsymbol{x}_{j}=\sum_{j=1}^{m} \boldsymbol{x}_{j}=1
\end{aligned}
$$

Due to the matrix form (3) of the operator (1), we can calculate the ergodic coefficient of the operator (1). The ergodic coefficient of the operator (1) is strictly less than one. Therefore, the trajectory of the operator (1) starting from an interior point $\boldsymbol{x}^{(0)} \in \operatorname{int} \boldsymbol{S}^{\boldsymbol{m}-1}$ converges to the center $\boldsymbol{C}=\left(\frac{1}{\boldsymbol{m}}, \frac{1}{\boldsymbol{m}}, \ldots, \frac{1}{\boldsymbol{m}}\right)$ of the simplex $\boldsymbol{S}^{\boldsymbol{m}-1}$. This completes the proof of Theorem. An interpretation of the mathematical results is as follows: if MAS is synchronized by a nonlinear rule given by (1) then a consensus can be always achieved. In other words, all subjective distributions of MAS eventually become equal.

\section{Conclusion}

In this paper, we have investigated a consensus problem for time-discrete MAS synchronized by a nonlinear rule. We proposed a new nonlinear protocol for MAS. Our protocol is a generalization of the classical DeGroot model. In our protocol, a consensus can be always achieved. It seems that, unlike a linear case, a consensus can be easily achieved a nonlinear case.

Acknowledgement: This work was supported by IIUM Endowment B Grant, EDW B 13-019-0904.

\section{References}

Bauso, D., Giarre, L. \& Pesenti, R. (2006). Non-linear protocols for optimal distributed consensus in networks of dynamic agents. Systems \& Control Letters, 55(11), 918-928.

Berger, R. L. (1981). A Necessary and Sufficient Condition for Reaching a Consensus Using DeGroot's Method. Journal of American Statistics Association, 76 (374), 415-418.

DeGroot, M. H. (1974). Reaching a consensus. Journal of American Statistics Association, 69(345), 118-121. 
Fax, J. A. \& Murray, R. M. (2004). Information flow and cooperative control of vehicle formations. IEEE Transactions on Automatic Control, 49(9), 1465-1476.

Ganikhodzhaev, R., Mukhamedov, F. \& Saburov, M. (2012). G-decompositions of matrices and related problems I. Linear Algebra and its Application, 436, 1344-1366.

Jadbabaie, A., Lin, J. \& Morse, S. (2003). Coordination of groups of mobile autonomous agents using nearest neighbor rules. IEEE Transactions on Automatic Control, 48, 988-1001.

Jadbabaie, A., Motee, N., \& Barahona, M. (2004). On the stability of the kuramoto model of coupled nonlinear oscillators. Proceedings of the American Control Conference, 4296-4301.

Kuramoto, Y. (1984). Chemical oscillations, waves, and turbulence. Springer, Berlin, Germany.

Lin, Z., Francis, B. \& Maggiore, M. (2007). State agreement for continuous-time coupled nonlinear systems. SIAM Journal on Control and Optimization, 46(1), 288-307.

Moreau, L. (2005). Stability of multi-agent systems with time dependent communication links. IEEE Transactions on Automatic Control, 50, 169-182.

Olfati-Saber, R. (2006). Flocking for multi-agent dynamic systems: Algorithms and theory. IEEE Transactions on Automatic Control, 51, 401-420.

Olfati-Saber, R., Fax, J. A. \& Murray, R. M. (2007).Consensus and cooperation in networked multi-agent systems. Proceedings of the IEEE, 95(1), 215-233.

Papachristodoulou, A. \& Jadbabaie, A. (2005). Synchronization in oscillator networks: Switching topologies and nonhomogeneous delays. Proceedings of the 44th IEEE Conference, 5692-5697.

Ren, W. \& Beard, R. W. (2008). Distributed Consensus in Multi-Vehicle Cooperative Control. London, U.K. Springer-Verlag.

Saburov, M. (2007). Onergodic theorem for quadratic stochastic operators. Dokl. Acad. Nauk. Rep. Uzb., 6, 8-11

Saburov, M. (2012). O regularity, transitivity, and Ergodic principle for quadratic stochastic Volterra operators. Proceedings of Academy of Science of Republic Uzbekistan, 3, 9-12.

Saburov, M. (2013). Some strange properties of quadratic stochastic Volterra operators. World Applied Sciences Journal, 21, 94-97

Strogatz, S. H. (2000). From kuramoto to Crawford: Exploring the onset of synchronization in populations of coupled oscillators. Physica $D, 143(1), 1-20$.

Strogatz, S. H. (2003). SYNC: The Emerging Science of Spontaneous Order. Hyperion Press, New York. 